\title{
Polyynes and Polycyclic Aromatic Molecules in C-rich Circumstellar Envelopes
}

\author{
Alain Omont \\ Institut d'Astrophysique de Paris, CNRS, 98bis, Bld Arago, F-75014 Paris, France
}

\section{Introduction}

The aim of this review is to discuss our knowledge on molecules in the circumstellar environment of evolved stars. In particular the presence and the behaviour of various kinds of molecules with several or many carbon atoms, in relation to C-rich dust, is considered.

Such objects include mainly: (i) circumstellar shells of AGB carbon stars, either visible (such as Y CVn) or infrared (such as IRC+10216 (CW Leo)); (ii) planetary nebulae (PNe, e.g. NGC 7027); (iii) pre-planetary nebulae (PPNe, also called post-AGB stars, such as CRL 2688 or the Red Rectangle), probably in an intermediate evolution stage between the two former classes. I will not discuss more peculiar classes, such as $\mathrm{R} \mathrm{CrB}$ stars and novae, for which very little is known about the presence of such molecular species.

Polyyne species are known from millimetre observations in AGB envelopes and PPNe, mostly in IRC+10216 (see Olofsson this volume). They are mainly cyanopolyynes $\left(\mathrm{HC}_{n} \mathrm{~N}\right)$ with $n=3,4, \ldots 11$, and $\mathrm{C}_{n}$ and $\mathrm{C}_{n} \mathrm{H}$ linear radicals, plus a few other cases $\left(\mathrm{C}_{4} \mathrm{H}_{2}, \mathrm{C}_{5}, \mathrm{C}_{3} \mathrm{~N}\right)$ (see Table 1). Observational results are discussed in Section 2: abundances, spatial distributions, etc.. Such information allows us to infer the basic features of their chemistry (Section 3).

In nearly all cases where their spatial distribution is known, the latter infers that they are formed mainly by photo-chemistry in the outer layers of the envelope, i.e. by chains of reactions induced by interstellar UV radiation. They thus have no direct relation with dust and their formation is surprising.

Polycyclic aromatic molecules (generally called PAHs) have been convincingly proposed as the carriers of the infrared bands observed in various interstellar and circumstellar environments. Aromatic species are presently not detected in AGB stars (see Hinkle this volume). On the other hand, PAHs are quite abundant in PNe and some PPNe (Section 4). They could thus be related to the evolution and processing of dust at the onset of the PN stage. Transition objects, PPNe, are therefore essential to elucidate this evolution. Their infrared spectroscopy, including dedicated studies, is discussed in Section 5. Together with the likely identification of PAH related species in the gas or in the dust, 
there remain several unidentified features. Indeed, there is no final conclusion whether PAHs are already present in the gas of AGB envelopes or they are generated at the onset of the PN stage. Because of lack of space, and also of evidence, I will not discuss the conjecture that $\mathrm{C}_{60}$ or its derivates could be present in such objects.

Table 1. Observed abundances in IRC+10216 (molecules denoted with a ${ }^{*}$ have only been detected in the infrared). $\mathrm{MgNC}$ has also recently been detected.

\begin{tabular}{|c|c|c|c|c|c|c|}
\hline$x(i) / H$ & & & IRC- & 216 & & \\
\hline $10^{-5}$ & $\begin{array}{l}\mathrm{C}_{2} \mathrm{H}_{2}^{*} \\
\mathrm{HCN}\end{array}$ & & & & & \\
\hline $10^{-6}$ & $\mathrm{NH}_{3}$ & $\mathrm{SiS}$ & CS & $\mathrm{HC}_{3} \mathrm{~N}$ & $\begin{array}{l}\mathrm{CN} \\
\mathrm{C}_{3}^{*} \\
\mathrm{C}_{2} \mathrm{H} \\
\mathrm{C}_{4} \mathrm{H}\end{array}$ & \\
\hline $10^{-7}$ & $\begin{array}{l}\mathrm{C}_{2} \mathrm{H}_{i}^{*} \\
\mathrm{CH}_{4}^{*}\end{array}$ & $\begin{array}{l}\mathrm{C}_{2} \mathrm{Si} \\
\mathrm{SiO}\end{array}$ & & $\mathrm{HC}_{5} \mathrm{~N}$ & $\begin{array}{l}\mathrm{C}_{3} \mathrm{~N} \\
\mathrm{C}_{5}^{*}\end{array}$ & \\
\hline $10^{-8}$ & $\begin{array}{l}\mathrm{CP} \\
\mathrm{CH}_{3} \mathrm{CN}\end{array}$ & $\begin{array}{l}\mathrm{SiN} \\
\mathrm{SiH}_{4}^{*} \mathrm{SiC}\end{array}$ & $\begin{array}{l}\mathrm{C}_{2} \mathrm{~S} \\
\mathrm{C}_{3} \mathrm{~S} \\
\end{array}$ & $\mathrm{HC}_{7} \mathrm{~N}$ & $\begin{array}{l}\mathrm{C}_{6} \mathrm{H} \\
\mathrm{C}_{3} \mathrm{H}_{2} \\
\mathrm{C}_{3} \mathrm{H} \\
\mathrm{C}_{3} \mathrm{H} \\
\mathrm{C}_{4} \mathrm{H}_{2}\end{array}$ & $\mathrm{ClAl}$ \\
\hline $10^{-0}$ & $\left(\mathrm{HCO}^{+}\right)$ & $\mathrm{C}_{4} \mathrm{Si}$ & $\mathrm{H}_{2} \mathrm{~S}$ & $\mathrm{HC}_{2} \mathrm{~N}$ & & $\begin{array}{l}\mathrm{ClNa} \\
\mathrm{ClK}\end{array}$ \\
\hline
\end{tabular}

\section{Observations of polyynes in circumstellar envelopes}

\subsection{Detected molecules and abundances in IRC+10216 (Table 1)}

Cyanopolyynes $\left(\mathrm{HC}_{2 n} \mathrm{CN}\right)$ were the first carbon chains detected in space. Many were first detected in IRC+10216. $\mathrm{HC}_{3} \mathrm{~N}, \mathrm{HC}_{5} \mathrm{~N}, \mathrm{HC}_{7} \mathrm{~N}, \mathrm{HC}_{9} \mathrm{~N}$ and $\mathrm{HC}_{11} \mathrm{~N}$ are now known. Their abundances do not decrease very rapidly with $n$. Typically the ratio

$$
H C_{2 n} C N / H_{2 n+2} C N \sim 3
$$

However, they are more difficult to detect for large $n$. Their very small rotation constant $B$ makes many rotational levels populated with the typical rotation 
temperatures $\sim 10-25 \mathrm{~K}$ and brings detectable lines into the centimetre range. The line intensities rapidly decrease despite the increase of the electric dipole momentum. They also have low vibrational levels allowing the detection of rotational lines of $\nu_{6}$ and $\nu_{7}$ states of $\mathrm{HC}_{3} \mathrm{~N}$.

Pure hydrocarbon chains, $H C_{2 n} H$, have not been detected. They have no permanent electric dipole momentum, and hence no radio spectrum. However, there is an infrared limit for the abundance of $\mathrm{HC}_{4} \mathrm{H}$ (see Hinkle this volume). Note that millimetre lines of its isomer $\mathrm{H}_{2} \mathrm{C}_{4}$ have been detected.

$C_{2 n} H$ radicals, with $\mathrm{n}=2,3\left(\mathrm{C}_{4} \mathrm{H}, \mathrm{C}_{6} \mathrm{H}\right)$ are quite abundant. They have several electronic levels of comparable low energy. $\mathrm{C}_{4} \mathrm{H}$ (and $C_{2} \mathrm{H}$ ) have a ${ }^{2} \Sigma$ ground state with a low ${ }^{2} \Pi$ state. The situation is reversed for the higher members such as $\mathrm{C}_{6} \mathrm{H}$ which have a ${ }^{2} \Pi$ ground state (Pauzat et al. 1989). This made difficult the identification of $\mathrm{C}_{6} \mathrm{H}$ in IRC+10216 (Guelin et al. 1987). These radicals have large electric dipole momenta. Rotation transitions of vibrationally excited $\mathrm{C}_{4} \mathrm{H}$ are easily detected in IRC+10216 where it is exceptionally abundant and extended. This could be related to the likely enhancement of the line strengths of vibration transitions by vibronic coupling.

$\mathrm{C}_{3} \mathrm{H}$ and $\mathrm{C}_{5} \mathrm{H}$ are also detected with the expected ${ }^{2} \Pi$ ground state, but with much lower abundances (Table 1).

Pure carbon chains, $C_{n}$, can be observed only by their infrared transitions (see Hinkle this volume). $\mathrm{C}_{3}$ and $\mathrm{C}_{5}$ have been detected with fairly large abundances in IRC+10216.

Several chain radicals with hetero-atoms have been found in IRC+10216: $\mathrm{C}_{3} \mathrm{~N}$ has a relatively large abundance, but the higher members $\mathrm{C}_{2 n+1} \mathrm{~N}$ are not detected, probably because of the smallness of their electric dipole momentum (Pauzat et al. 1991). $\mathrm{C}_{3} \mathrm{~S}$ (and $\mathrm{C}_{2} \mathrm{~S}$ ) and $\mathrm{C}_{4} \mathrm{Si}$ are also found with low abundances.

\subsection{Spatial Distribution in IRC +10216}

The determination of the position in the envelope of the observed molecules is essential to analyze the chemical processes. To do this clearly requires the telescope beam to be smaller than the extension of the molecular envelope. The exceptionally close proximity of IRC +10216 for a source with a large mass-loss rate (see Olofsson this volume) yields very large angular diameters (20-60"). Accordingly, its envelope is resolved with the small beams of large millimetre single dishes. For instance the beam of the IRAM $30 \mathrm{~m}$ telescope is 12 " at $1.3 \mathrm{~mm}$. Several studies of the extensions of various molecules have been carried out with single dishes. Indeed, the shape of the profile observed in the center of the source provides by itself a useful information on the extension of the molecule (see Olofsson). However, the large millimetre interferometers are unravelled to 
give complete details on the molecular distributions. The spatial distribution of most important molecules is now well studied in IRC+ 10216 (see Olofsson this volume). The most important contribution has been first provided by Bieging, Nguyen-Q-Rieu and collaborators with the Hat Creek interferometer since several years (Bieging \& Nguyen-Q-Rieu 1988, Nguyen-Q-Rieu \& Bieging 1990, Dayal \& Bieging 1993, Bieging \& Taffala 1993). The IRAM interferometer is now the most sensitive instrument and is making systematic studies of many molecular lines (see e.g. Guélin 1993).

All polyyne species studied $\left(\mathrm{HC}_{3} \mathrm{~N}, \mathrm{HC}_{5} \mathrm{~N}, \mathrm{C}_{4} \mathrm{H}, \mathrm{C}_{3} \mathrm{~N}\right)$ display quite characteristic structures of hollow shells (slightly clumpy, Fig. 1). It is quite likely that this is also true for all the other polyynes whose weaker lines have profiles characteristic of a large extension, but are not yet completely studied with interferometer mapping. It is thus proved that most detected polyyne molecules are synthesized in the outer shells of the envelope permeated by the interstellar UV and are not generated in the dense regions close to the star (see Section 3).
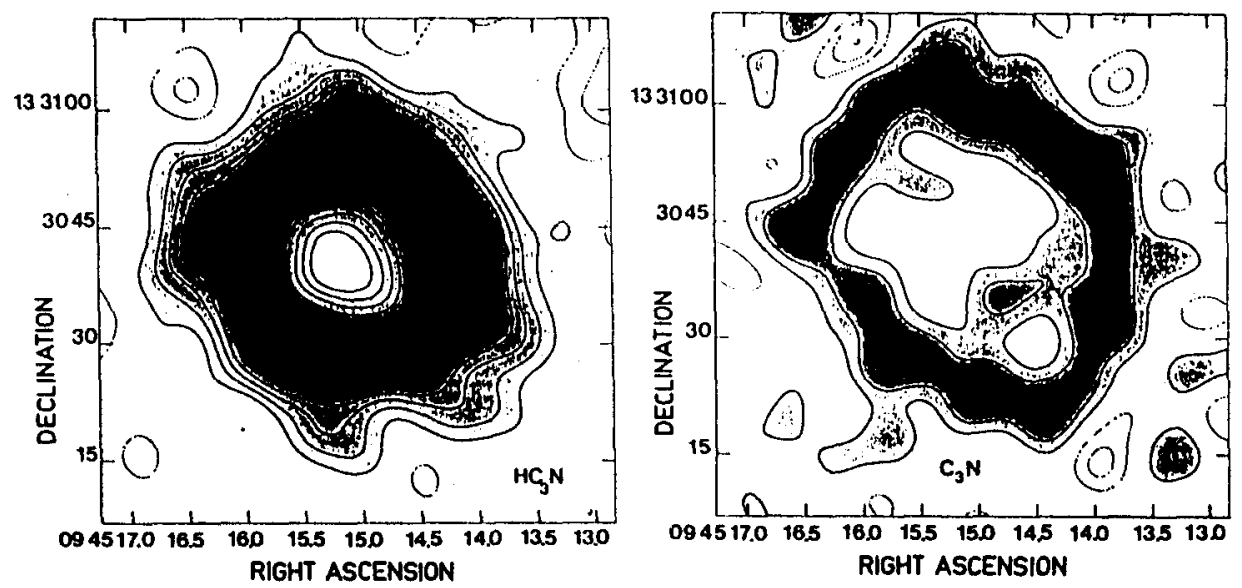

Fig. 1. Interferometer maps of $3 \mathrm{~mm}$ lines of $\mathrm{HC}_{3} \mathrm{~N}$ and $\mathrm{C}_{3} \mathrm{~N}$ in $\mathrm{IRC}+10216$ by Bieging \& Taffala (1993)

\subsection{Polyynes in Envelopes of Other Stars}

The information is much more meagre on molecules in stars other than IRC+10216, since all millimetre molecular lines are at least 10 times weaker (see Olofsson this volume). However, the strongest lines $\left(\mathrm{C}_{4} \mathrm{H}, \mathrm{C}_{3} \mathrm{~N}, \mathrm{HC}_{5} \mathrm{~N}\right.$ and especially $\mathrm{HC}_{3} \mathrm{~N}$ ) are easily detected in 10-20 C-rich envelopes with large mass-loss rates (a few $10^{-6}$ - a few $10^{-5} M_{\odot} / \mathrm{yr}$ ) comparable to that of IRC+10216. The most studied sources are CIT 6 (RW LMi), IRAS (15194-5115) (Nyman et al. 1993), AFGL 3068, IRC+40540 (LP And), IRC+10401 (Cernicharo et al. 1994), etc... 
There are no striking differences in the abundances compared to IRC+10216. In particular the ratio of the line intensities $\mathrm{HC}_{3} \mathrm{~N} / \mathrm{HCN}$ is remarkably constant (Bujarrabal et al. 1994). However, in sources with large excesses of HCN, such as IRC+10401, the abundances of $\mathrm{HC}_{3} \mathrm{~N}, \mathrm{HC}_{5} \mathrm{~N}$ and $\mathrm{C}_{4} \mathrm{H}$ are also probably an order of magnitude larger than in IRC+10216. On the other hand the abundances of $\mathrm{HC}_{3} \mathrm{~N}$ and especially the ratio $\mathrm{HC}_{3} \mathrm{~N} / \mathrm{HCN}$ are quite small in $\mathrm{C}-$ rich envelopes with low mass-loss rates such as $\mathrm{Y} \mathrm{CVn}$. $\mathrm{HC}_{3} \mathrm{~N}$ has never been detected in O-rich stars (Bujarrabal et al. 1994).

In C-rich pre-planetary nebulae the abundance of $\mathrm{HC}_{3} \mathrm{~N}$ (and of $\mathrm{C}_{4} \mathrm{H}, \mathrm{HC}_{5} \mathrm{~N}$ ) is also generally comparable to that of IRC +10216 , although somewhat smaller (Henkel et al. 1994, Lucas et al. 1994). The latter authors have performed a detailed study of many lines in AFGL2688 (CRL2688, the Egg Nebula); $\mathrm{HC}_{3} \mathrm{~N}$, but not $\mathrm{C}_{4} \mathrm{H}$, displays modest high velocity wings. $\mathrm{HC}_{3} \mathrm{~N}$ has been mapped with the Hat Creek inteferometer (Nguyen-Q-Rieu \& Bieging 1990), but not with enough sensitivity to derive detailed information about its spatial distribution. However, cyanopolyynes display important pecularities in this source as well as in the molecular envelope of the extremely young planetary nebula AFGL 618. AFGL 2688 is the prototype of bipolar nebulae. The VLA map of $\mathrm{HC}_{7} \mathrm{~N}$ at $1.4 \mathrm{~cm}$ by Nguyen-Q-Rieu et al. 1986 (Fig. 2) shows that its emission is relatively strong and extremely extended ( $\mathrm{r} \sim 20-30$ ") compared to the size of the emission of the other molecules such as $\mathrm{HCN}$ or $\mathrm{NH}_{3}(\mathrm{r} \leq 10$ "). This extension is at maximum in the direction of the axis of the nebula and of its bipolar flow, but it is also important in the perpendicular direction. Jura \& Kroto (1990) have proposed that $\mathrm{HC}_{7} \mathrm{~N}$, as well as other long chains, are generated in grain-grain collisions of grains accelerated by the radiation pressure of visible radiation. A millimetre map of $\mathrm{HC}_{5} \mathrm{~N}$ made using the IRAM interferometer (Lucas et al. 1994) does not display such a large extension. However, this could only reflect the difficulty of exciting millimetre lines far from the star. The modest extension of this $\mathrm{HC}_{5} \mathrm{~N}$ emission is again mostly axial, but with also a non axial feature at an intermediate velocity.

AFGL 618 (CRL 618) is indeed a nascent planetary nebula, with a very small and expanding ionized region inside a massive molecular envelope. The millimetre $\mathrm{HC}_{3} \mathrm{~N}$ lines are quite prominent and have a very large rotation temperature. Even the rotation lines of vibrationaly excited $\mathrm{HC}_{3} \mathrm{~N}$ are quite strong.

On the other hand, $\mathrm{HC}_{3} \mathrm{~N}$ has never been detected in any other, more regular, planetary nebula, even the very young one NGC 7027, despite active searches (Cox et al. 1994).

\section{Chemistry of Polyynes in IRC+10216}

The spatial distribution of polyynes in IRC+10216 clearly points to generation by photochemistry. Indeed, the understanding of their formation was probably the main challenge of circumstellar chemistry and the results already obtained are a major achievement. The general features of circumstellar chemistry are now well established (see e.g. reviews by Glassgold \& Huggins 1988, Millar 


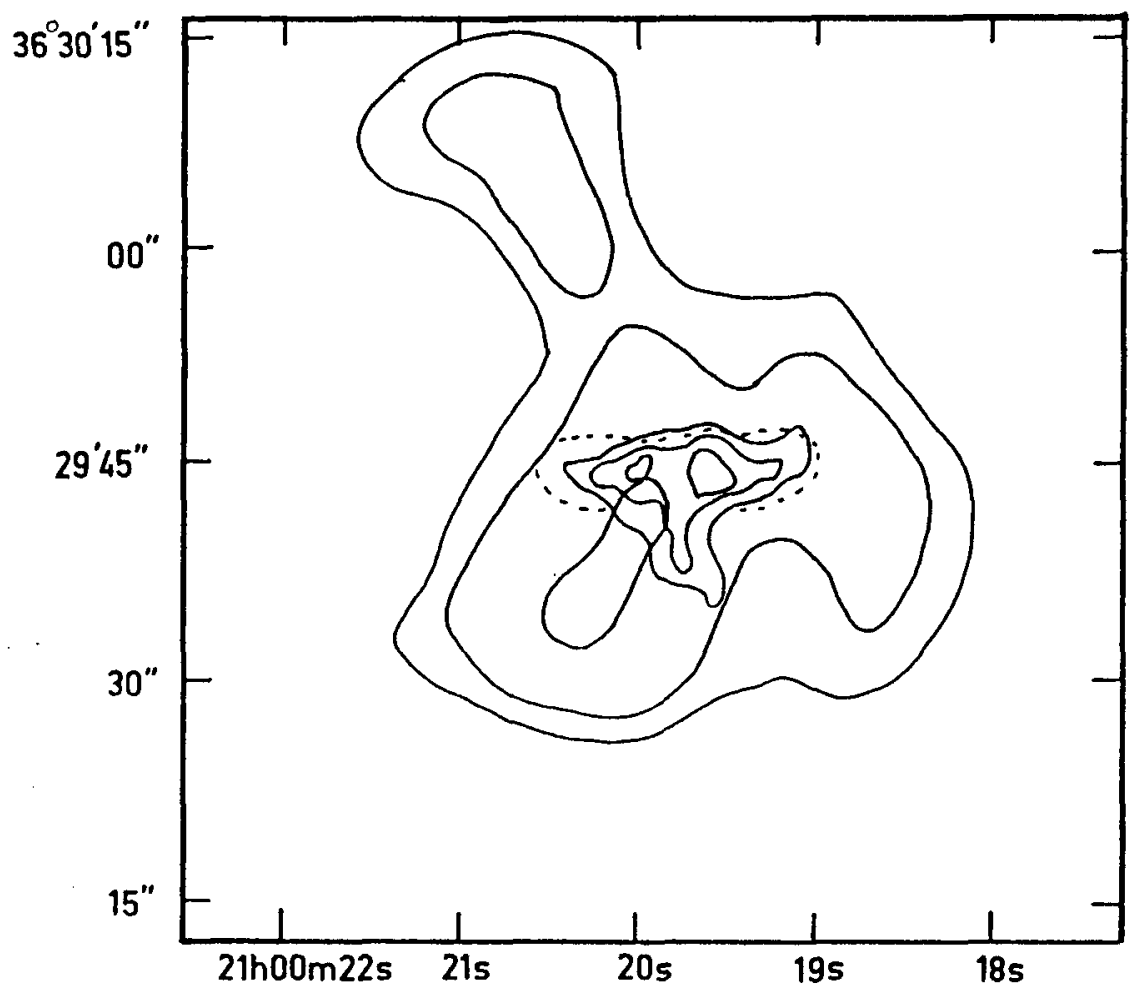

Fig. 2. VLA interferometer map of $1.4 \mathrm{~cm}$ lines of $\mathrm{HC}_{7} \mathrm{~N}$ (outer contours) and $\mathrm{NH}_{3}$ (inner contours) by Nguyen-Q-Rieu et al. (1986) (from Jura \& Kroto 1990)

1988, Omont 1990). Thermodynamical chemical equilibrium is achieved only in the deep inner layers, dense and warm, close to the photosphere. Most stable molecules, such as $\mathrm{H}_{2}, \mathrm{CO}, \mathrm{N}_{2}, \mathrm{C}_{2} \mathrm{H}_{2}, \mathrm{HCN}, \mathrm{CS}, \mathrm{SiS}, \mathrm{SiO}$, are generated there. However, calculations of equilibrium abundances (see e.g. Tejero \& Cernicharo 1991) derive a relatively small abundance for $\mathrm{HC}_{3} \mathrm{~N}$ and much smaller ones for other polyynes.

The chemistry is certainly complex and poorly understood in the intermediate layers where dust and the circumstellar wind are generated. Small carbon chains appear as possible initial steps in the nucleation of C-rich dust. However, given the observed shell distribution of polyynes, their abundance must remain quite small at the end of dust formation. Photochemistry dominates the chemistry in the outer layers which are not shielded against the penetration of the interstellar UV radiation. The latter first destroys the stable molecules, such as $\mathrm{CO}$, $\mathrm{C}_{2} \mathrm{H}_{2}, \mathrm{HCN}$, etc, transported by the expansion from the inner layers. Thus, the photodestruction products, radicals such as $\mathrm{C}_{2} \mathrm{H}$ and $\mathrm{CN}$, atoms and ions such as $\mathrm{C}_{2} \mathrm{H}_{2}^{+}$and $\mathrm{C}^{+}$, can react with abundant species to build up more and more 
complex polyyne chains.

Detailed modelling of such photochemistry has been developed by Glassgold and collaborators, and Millar and collaborators. The input parameters are the envelope parameters (mass-loss rate, expansion velocity, temperature, etc), the initial abundances of stable molecules such as $\mathrm{CO}, \mathrm{C}_{2} \mathrm{H}_{2}, \mathrm{HCN}$ coming from the inner shells, the UV intensity and the reaction and photodestruction rates. One has to model the transfer of the UV radiation coming from the interstellar medium and progressively attenuated by dust in the outer layers of the envelope. A part of the photodissociation rates can be reasonably well estimated; $\mathrm{CO}$ needs a special elaborate treatment (Mamon et al. 1988). However, most of the photodestruction rates of unstable species can only be roughly guessed. Ion reaction rates are reasonably well known for small species. A typical proposed reaction is (Glassgold et al. 1986, 1987)

$$
\mathrm{C}_{2} \mathrm{H}_{2}^{+}+\mathrm{HCN} \rightarrow \mathrm{H}_{2} \mathrm{C}_{3} \mathrm{~N}^{+}+\mathrm{H}
$$

and then $\mathrm{H}_{2} \mathrm{C}_{3} \mathrm{~N}^{+}$could yield $\mathrm{HC}_{3} \mathrm{~N}$ by dissociative recombination. However, it is now recognised that such ion reactions are not efficient enough to account for the large amount of observed polyynes. Radical reactions are more effective given the large reaction rates at low temperature (Nejad \& Millar 1987, 1988). For instance, possible reactions are

$$
\begin{aligned}
& \mathrm{CN}+\mathrm{C}_{2} \mathrm{H}_{2} \rightarrow \mathrm{HC}_{3} \mathrm{~N}+\mathrm{H} \\
& \mathrm{C}_{3} \mathrm{~N}+\mathrm{C}_{2} \mathrm{H}_{2} \rightarrow \mathrm{HC}_{5} \mathrm{~N}+\mathrm{H}
\end{aligned}
$$

If early models still had difficulties in accounting for the long chains, the last model of Cherchneff et al. (1993), Cherchneff \& Glassgold (1993) gives satisfactory results for the first cyanopolyynes, $\mathrm{C}_{4} \mathrm{H}$, etc, with the large reaction rates now admitted for polar molecules at very low temperature.

In conclusion, although many uncertainties remain (in particular in the detailed rates) there is very good agreement between the observed abundances and their distribution. The results of theoretical chemical modelling demonstrates that most, if not all, polyynes observed in IRC+10216 are synthesized by photochemistry in the outer layers from small molecules such as $\mathrm{C}_{2} \mathrm{H}_{2}, \mathrm{HCN}$, etc. The situation is likely to be the same in similar AGB envelopes. It is indeed slightly paradoxal that the observed polyynes have no direct relation with $\mathrm{C}$-rich dust, while they have been proposed as logical intermediates in dust nucleation, as well as natural products of grain-grain collisions (Jura \& Kroto 1990).

\section{Polycyclic Aromatic Molecules and Circumstellar Envelopes}

Polycyclic aromatic hydrocarbons (PAHs) are also a quite natural and stable form of small carbon clusters in the hydrogen rich environment of the interstellar and circumstellar media, probably playing an important role in the nucleation 
of circumstellar grains (see Sedlmayr this volume). Complex mixtures of such PAHs have been convincingly proposed as the carriers of the set of characteristic infrared bands at 3.3,6.2, 7.7, 8.6, $11.3 \mu \mathrm{m}$ observed (Fig. 3) in various interstellar and circumstellar sources exposed to UV radiation (see e.g. Léger \& Puget 1984, Allamandola et al. 1985, Puget \& Léger 1989 and references therein).

The molecular envelopes of young planetary nebulae, such as NGC 7027, and of pre-planetary nebulae with early type stars, such as the Red Rectangle (HD 44179), are major sources of PAHs.

The "canonical model" of PAH IR emission induced by UV radiation (see references above), explains the very large observed vibrational temperature, practically independent of the UV intensity: free-flying PAHs, with 20-100 atoms, are small enough systems to be heated to $500-1000 \mathrm{~K}$ by a single UV photon. The main arguments which have lead to this interpretation are their very large stability, the high specificity of some bands $(6.2 \mu \mathrm{m})$ characteristic of aromatic compounds and the properties of the observed vibration temperature not achievable in conventional grains. However, several problems remain concerning their stability in astrophysical conditions, as well as their degree of ionization and of de-hydrogenation and the related spectral characteristics.

In circumstellar envelopes, PAH emission is quite strong in PNe and warm $\mathrm{PPNe}$, but they are not observed in AGB stars. This raises interesting questions about their generation and their excitation. Many details are known on PAH emission in the strongest sources such as NGC 7027 and the Red Rectangle.

NGC 7027 is a PN with a very hot star emitting very strong UV radiation. A dense expanding ionized central region coexists with a massive outer molecular envelope partially permeated by the UV radiation (Cox et al. 1994). The PAHs probably reside mostly at the interface between the ionized and molecular gas. PAH fluorescence is clearly induced by the strong UV radiation. It is quite possible that the PAHs are generated at the onset of the PN stage by some mechanism such as destruction and processing of dust in such a violent environment. However, any detailed evidence is still lacking, and they could as well have been present at early stages and be just revealed by the strong UV shining.

The situation has some similarities in the Red Rectangle (HD 44179) but with important pecularities. This is a PPN with a warm star (Ao), but not hot enough to ionize the circumstellar gas. There is some indication of a circumstellar disk, which could explain that the $\mathrm{CO}$ millimeter lines are very narrow and also very weak because of its likely photodissociation. Radiation pressure on dust in such a disk could also be at the origin of the strange photospheric abundances with a strong underabundance of iron and other refractory elements. This nebula has a very strong and peculiar red fluorescence with a molecular band structure reminiscent of unidentified visible diffuse interstellar bands, and possibly associated with PAHs.

There is presently no evidence for either the presence or absence of PAHs in ordinary AGB circumstellar envelopes. The lack of UV and even visible radiation would explain the absence of infrared bands even in the presence of PAHs. It 


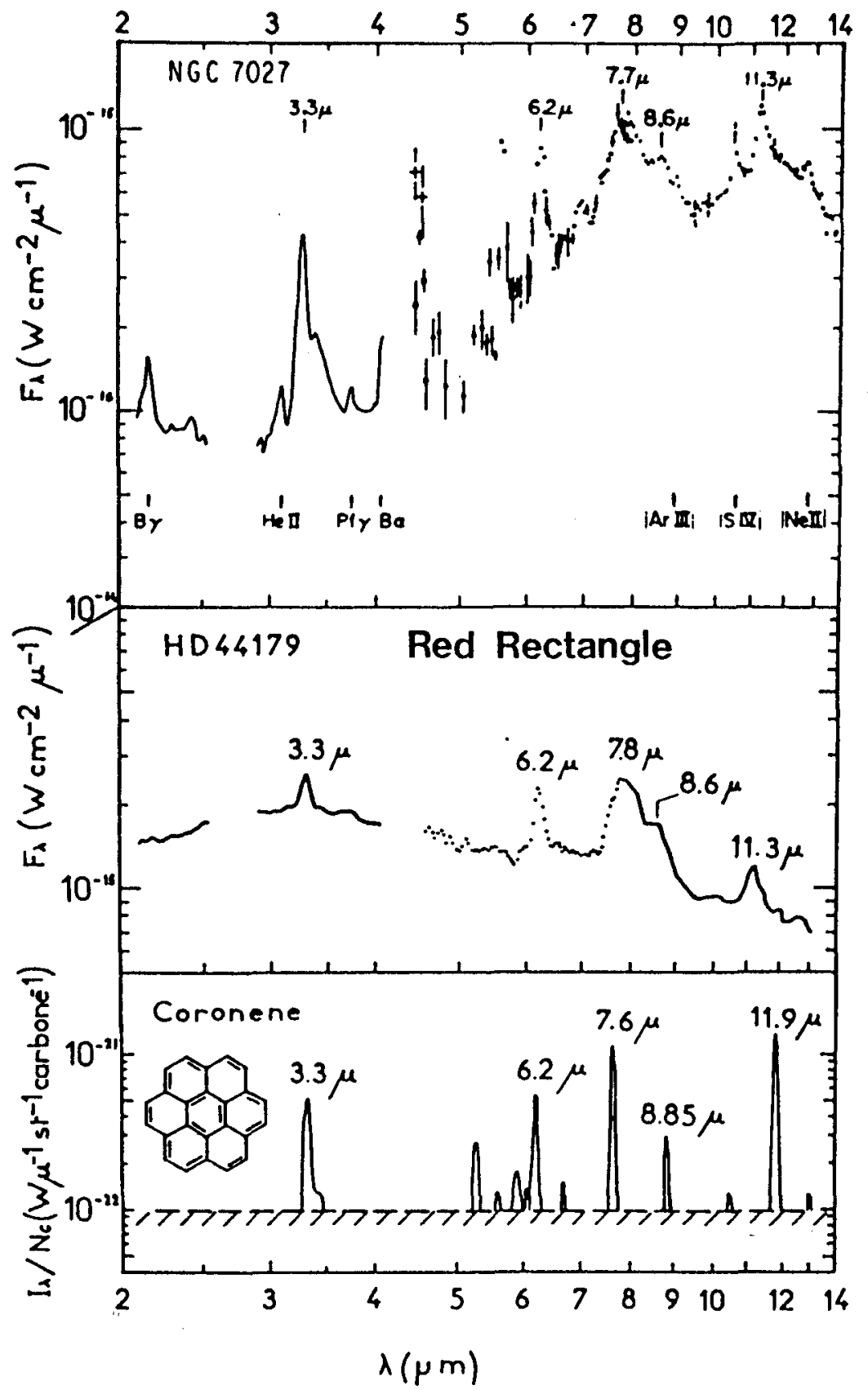

Fig. 3. Infrared features observed in NGC 7027 and HD 44179 (Red Rectangle) compared to a synthetic spectrum of coronene, from Léger \& Puget (1984). 
would be very difficult to see them in absorption, as well as to detect their radio rotational lines. Since they are likely intermediates in the formation of C-rich dust in the inner shells, they could well survive in sufficient amount (a few percents of the total carbon) in the whole envelope. However, a simple model of dust formation (Cherchneff et al. 1993) leads to quite small amounts of PAHs in the outer envelope.

An alternative, not unlikely possibility would be that they are formed, or just desorbed, from $\mathrm{C}$-rich grains at the onset of the PPN-PN stage. The properties of dust would then rapidly evolve and a natural question is: are there spectral features related to such transitory stages of dust in PPNe? A related question is: are there spectral features in C-rich envelopes related to PAHs either free, or incorporated in dust (i.e. any evidence of hydrogenated amorphous carbon (HAC) which is a mixture of polycyclic carbon clusters partially hydrogenated) ?

\section{Infrared Spectroscopy of C-rich Circumstellar Dust}

\subsection{Spectra of Ordinary AGB Envelopes}

They are relatively featureless because the main constituent of dust is solid carbon which has no prominent spectral feature. However, there are several strong and characteristic features:

- In the near infrared there is a strong absorption band of the gas at $3.4 \mu \mathrm{m}$ due mainly to $\mathrm{C}_{2} \mathrm{H}_{2}$ and $\mathrm{HCN}$, as well as a few other weaker features at longer wavelengths in some cases.

- The middle infrared is very well documented by "Low Resolution Spectra" (LRS) of the IRAS satellite between 7.5 and $23 \mu \mathrm{m}$ which contain several hundred spectra of C-rich envelopes. Most of them display a strong characteristic band at $11.4 \mu \mathrm{m}$ attributed to solid $\mathrm{SiC}$.

In addition, there is quite often a less obvious feature at the lower LRS limit, which could be either an emission at $8.5 \mu \mathrm{m}$ or absorption at $7.5 \mu \mathrm{m}$ (gas bands ?) (see e.g. Omont et al. 1993) and references therein).

- In the far infrared, there is a strong and broad feature at about $30 \mu \mathrm{m}$, when the mass-loss is large enough ( $\geq$ a few $10^{-6} M_{\odot} / y r$ ) (Forrest et al. 1981). Although it has been attributed to solid MgS (Goebel \& Moseley 1985), its origin is still discussed. A similar feature is also often present in PNe and PPNe (Section 5.3).

\subsection{Infrared Spectroscopy of cold C-rich PPNe}

We have already discussed the case of "warm" PPNe such as the Red Rectangle (Section 4). In typical "cold" PPNe, such as the Egg Nebula (AFGL 2688), there is no warm dust because the expanding envelope is completely detached from the star with a nearly empty central cavity around the star. Accordingly, the IR spectrum has its maximum in the far infrared, beyond $20 \mu \mathrm{m}$ (Fig. 4). As seen 
in this figure, the IRAS LRS spectra are mainly of two types: a good part of them, including AFGL 2688 and other thick envelopes, are nearly featureless.
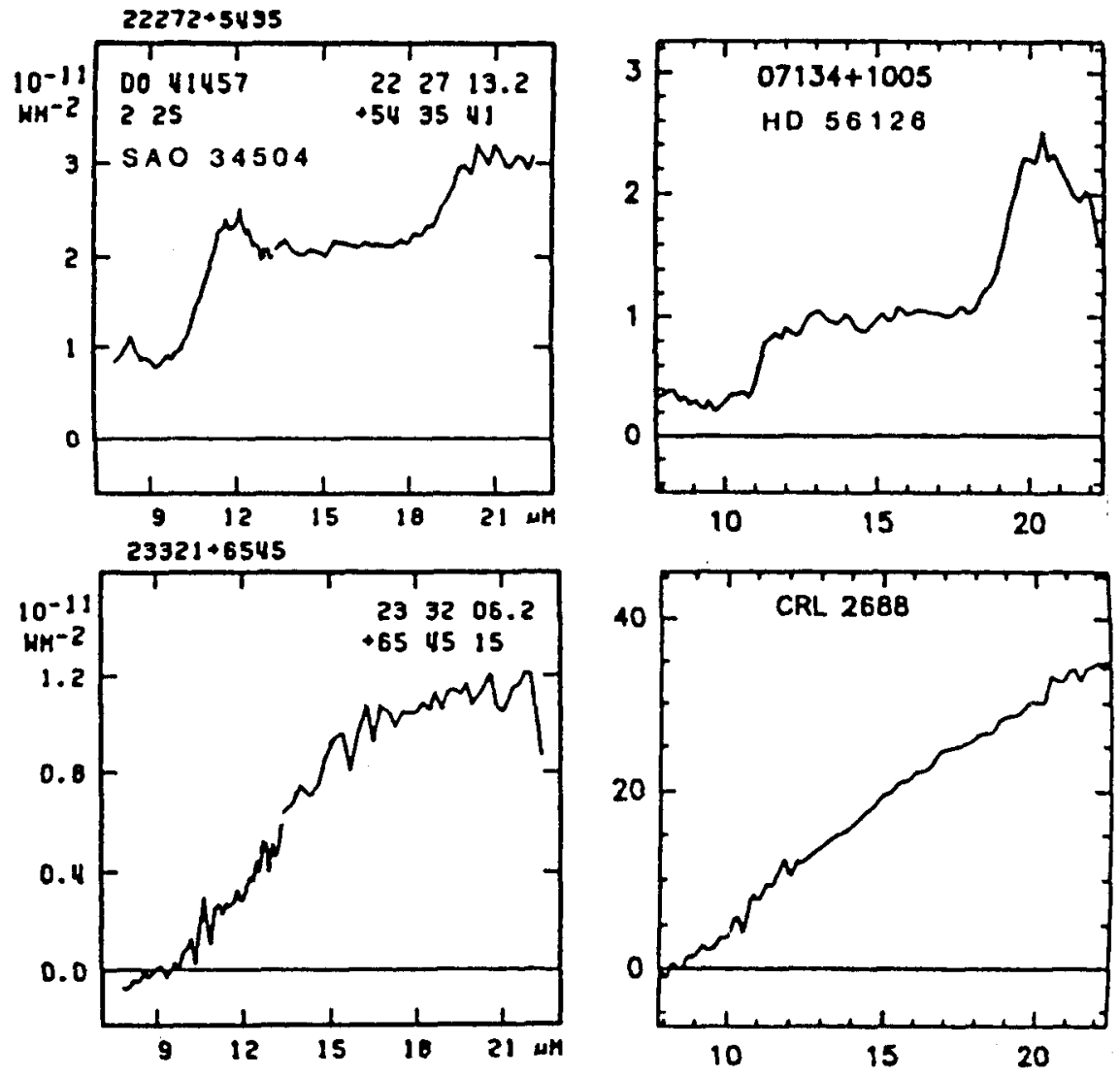

Fig. 4. IRAS Low Resolution Spectra (1986) of C-rich pre-planetary nebulae with and without an emission feature at $21 \mu \mathrm{m}$.

On the other hand, the LRS spectra of other sources display two strong features: a broad band at $21 \mu \mathrm{m}$ and a plateau between 12 and $15 \mu \mathrm{m}$ (Kwok et al. 1989). They have a relatively thin envelope so that they emit comparable amounts of energy in the visible and the far infrared. Airborne spectroscopy of the 5-8 $\mu \mathrm{m}$ range (Buss et al. 1990) shows several features reminiscent of those attributed to PAHs, but somewhat different and weaker when compared to strong classical PAH sources (Fig. 5). In addition, the $3.3 \mu \mathrm{m}$ "PAH" feature has also been detected in some sources, as well as other features at about $3.4 \mu \mathrm{m}$. All these characteristics have lead Buss et al. to propose that they are 
emitted by some carbonaceous compound containing hydrogenated amorphous carbon (HAC). It should be noted that similar, although weaker, features are also present in AFGL 2688.

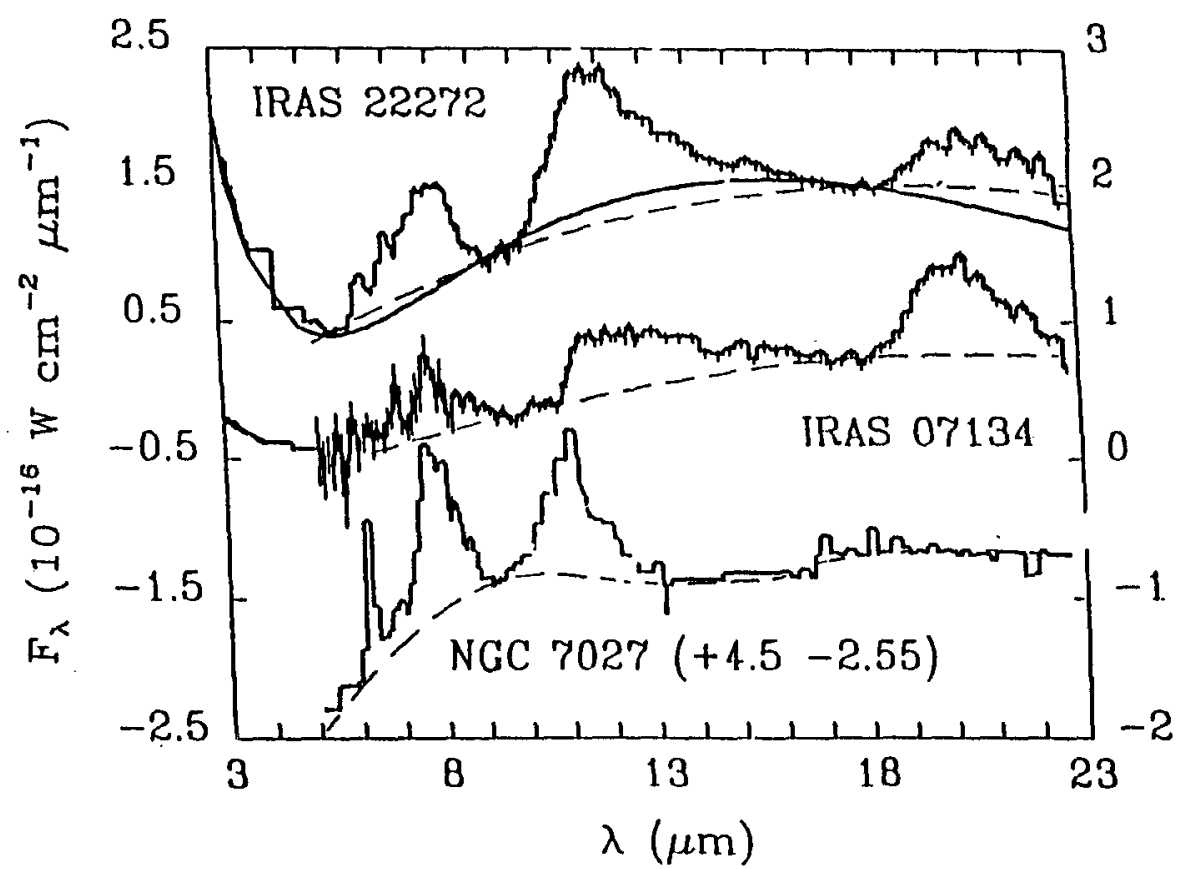

Fig. 5. 5-23 $\mu \mathrm{m}$ spectrum of two C-rich pre-planetary nebulae displaying the $21 \mu \mathrm{m}$ emission feature, compared to the spectrum of the PAH source NGC 7027 (from Buss et al. 1990).

The origin of $21 \mu \mathrm{m}$ band is not yet entirely clear. It seems likely, as suggested by Buss et al. (1990), that it is associated with some kind of carbonaceous compound. Such materials frequently emit at $11-15 \mu \mathrm{m}$, so that there is no difficulty to explain the companion feature observed in this range. However, no compound is presently known with such a strong emission at $21 \mu \mathrm{m}$ and the other observed spectral characteristics (see e.g. Omont et al. 1994 and references therein). Many of the PAHs, such as naphtalene, have strong bands in the 19$23 \mu \mathrm{m}$ range, but they have also other bands which are not observed. It would obviously be quite interesting to identify the carrier of the $21 \mu \mathrm{m}$ band. It should be a transient species, characteristic of dust processing in these PPNe, since the feature is not observed in their AGB progenitors, neither in the following $\mathrm{PNe}$ stages. Omont et al. (1994) have recently extended the spectroscopy of these $\mathrm{C}$-rich PPNe up to $50 \mu \mathrm{m}$ by airborne KAO observations. There is no other relatively narrow feature such as the one at $21 \mu \mathrm{m}$. 
IRAS $22272+5435$
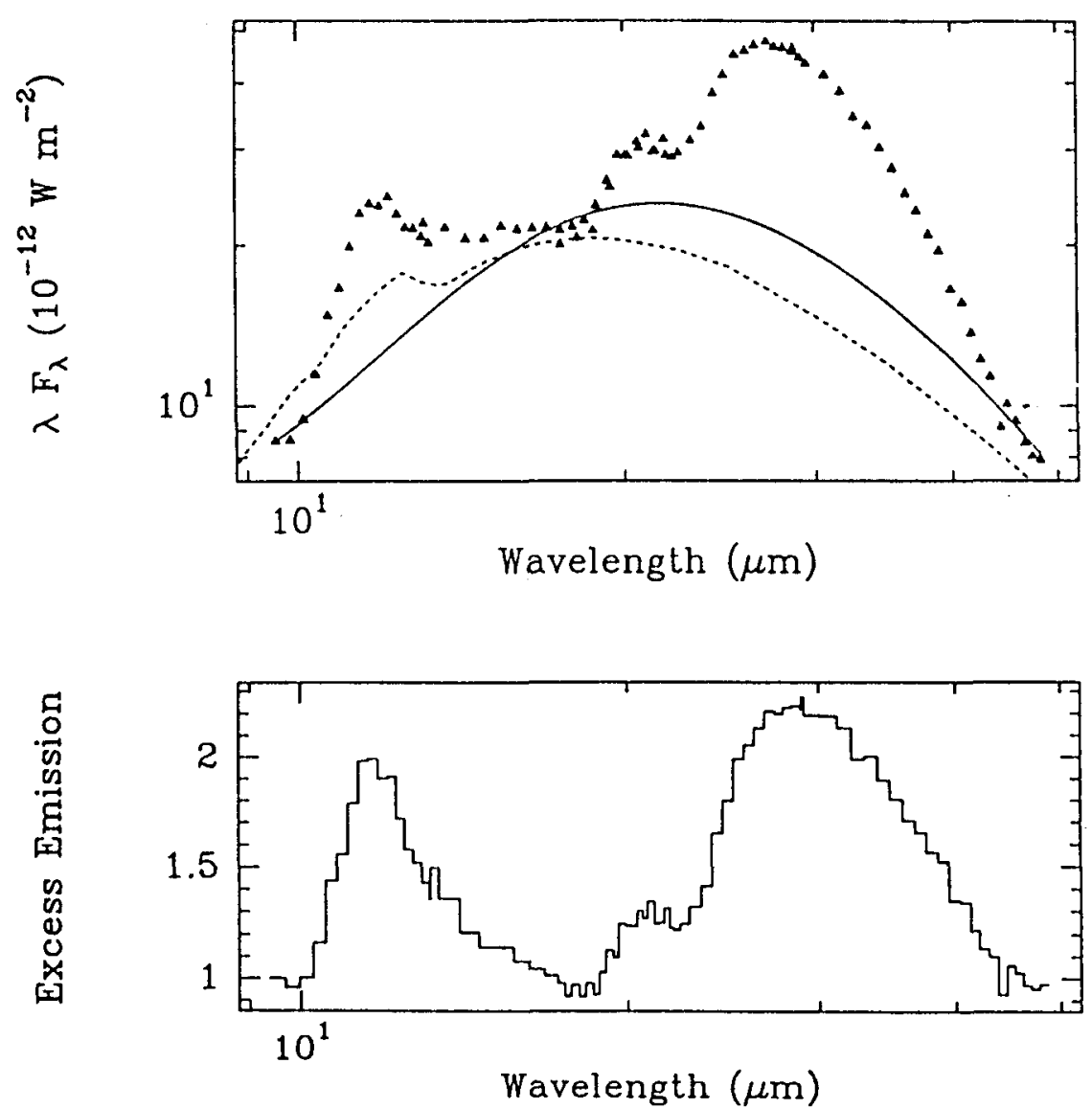

Fig. 6. Composite for infrared spectrum of IRAS $22272+5435$ from Omont et al. (1994). The continuous and dashed curves represent two different estimates of the background emission.

However, most sources display a very broad excess of emission between 25 and $45 \mu \mathrm{m}$ (Fig. 6). The intensity of this emission is quite variable following the source and apparently does not correlate with the strength of the $21 \mu \mathrm{m}$ band. This $30 \mu \mathrm{m}$ emission appears similar to the one observed in AGB carbon stars with large mass-loss (Forrest et al. 1981) as well as in some young planetary nebulae (Moseley \& Silverberg 1986, Glaccum 1989). Both of the latter cases have been attributed to MgS by Goebel \& Moseley (1985). However, the remarkable strength of the emission in sources such as IRAS 22272 and the PN IC 418 casts some doubt on this. Further laboratory data on $\mathrm{MgS}$ are required to check this. Other possible explanations should also be explored, such as the emission of metals ( $\mathrm{Fe}, \mathrm{Mg}$...) attached to aromatic compounds (Chaudret et al. 
1991, Kroto \& Jura 1992).

Acknowledgments. I am glad to thank Mrs M.C. Pantalacci for the edition of this paper.

\section{References}

Allamandola L.J., Tielens A.G.G.M., Barker J.R., 1985, Astrophys. J. 290, L25

Bieging J.H., Taffala M., 1993, Astrophys. J., 105, 576

Bieging J.H. and Nguyen-Q-Rieu, 1988, Astrophys. J., 329, L107

Bujarrabal V., Fuente A., Omont A. 1994, Astron. Astrophys., in press

Buss Jr R.H., Cohen M., Tielens A.G.G.M., Werner M.W., Bregman J.O., Witteborn F.C., Rank D., Sandford S.A., 1990, Astrophys. J. Lett., 365, L23

Cernicharo J., Fuente A., Omont A. 1994, in preparation

Chaudret B., Lebeuze A., Rabaa H., Saillard J.Y., Serra G., 1991, New J. Chem., 15, 791

Cherchneff I., Barker J.R., 1992, Astrophys. J., 394, 703

Cherchneff I., Barker J.R., Tielens A.G.G.M., 1992, Astrophys. J., 401, 269

Cherchneff I., Glassgold A.E., Mamon G.A., 1993, Astrophys. J., 410, 188

Cherchneff I., Glassgold, A.E., 1993, Astrophys. J. Lett. in press

Cox P., Guilloteau S., Bachiller R., Huggins P.J., Omont A., Forveille T., 1994, in preparation

Dayal A., Bieging J.H., 1993, Astrophys. J. Lett., 407, L37

Forrest W.S., Houck J.R., Mc Carthy J.F., 1981, Astrophys. J., 248, 195

Glaccum W.J., 1989, PhD Dissertation

Glassgold A.E., Lucas R. and Omont A., 1986, Astron. Astrophys., 157, 35

Glassgold A.E. and Huggins P.J., 1988, in "M-Type Stars", ed. by H.R. Johnson and F. Querci (NASA-CNRS), p.291

Goebel J.H., Moseley S.M., 1985, Astrophys. J. Lett., 290, L35

Guélin M., Cernicharo J., Kahane C., Gomez-Gonzalez J., Walmsley C.M., 1987, Astron. Astrophys., 175, L5

Guélin M. 1993, in "Circumstellar Media in the Late Stages of Stellar Evolution", ed. by R.E.S. Clegg

Henkel C., Cox P., Omont A. 1994, in preparation

Howe D.A. and Millar T.J., 1990, MNRAS, 244, 444

IRAS Team, LRS Atlas 1986, Low Resolution Spectra Atlas, Olnon F.M. and Raymond E., Astron. Astrophys. Supp., 65, 607

Jura M., Kroto H., 1990, Astrophys. J., 351, 222

Kroto M. and Jura M., 1992, Astr. Ap., 263, 275

Kwok S., Volk K.M., Hrivnak B.J., 1989, Astrophys. J. Lett., 345, L 51

Léger A., Puget J.L., 1984, Astron. Astrophys., 137, L5

Lucas R., Kahane C., Omont A. 1994, in preparation

Mamon G.A., Glassgold A.E. and Huggins P.J., 1988, Astrophys. J., 328, 797.

Millar T.J., 1988, In Rate Coefficients in Astrochemistry, ed. by T.J. Millar and D.A. Williams (Kluwer Academic Pub.), p.287

Moseley S.H., Silverberg R.F., 1986, in: KAO Workshop, p. 233, (NASA)

Nejad L.A.M. and Millar T.J., 1987, Astron. Astrophys., 183, 279

Nejad L.A.M. and Millar T.J., 1988, MNRAS, 230, 79 
Nguyen Q Rieu, Winnberg A., Bujarrabal V., 1986, Astron. Astrophys., 165, 204 Nguyen-Q-Rieu, Bieging J.H., 1990, Astrophys. J., 359, 131

Nyman L.A., Olofsson M., Johansson L.E.B., Booth A.S., Carlström U., Wolstencroft R., 1993, Astron. Astrophys., 269, 377

Omont A., 1990 In "Chemistry in Space" ed. by M.Greenberg and V.Pironello (Kluwer, Dordrecht, 1990)

Omont A., Loup C, Forveille T., te Lintel Hekkert P., Habing H., Sivagnanam P., 1993, Astron. Astrophys., 267, 515

Omont A., Moseley S.H., Cox P., et al., 1994, to be submitted to Astrophys. J. Lett.

Pauzat F., Ellinger Y., 1989, Astrom. Astrophys., 216, 305

Pauzat F., Ellinger Y., Mc Lean A.D., 1991, Astrophys. J. Lett., 369, L13

Puget J.L., Léger A., 1989, Ann. Rev. Astron. Astrophys., 27, 161

Tejero J., Cernicharo J. 1991, Instituto Geografico Nacional, Madrid 